\title{
Paciente idosa portadora de doença aterosclerótica submetida à cineangiocoronariografia com achado incidental de origem e curso anômalos de artéria coronária esquerda: relato de caso
}

\author{
Patient elderly carrier of atherosclerotic disease undergoing coronary \\ angiography with incidental finding of origin and course of \\ anomalous left coronary artery: a case report
}

\author{
Luilson Geraldo Coelho Júnior ${ }^{1}$, Pedro Rodrigo Magalhães Negreiros de Almeida ${ }^{1}$, Wesley \\ Lobo Costa Junior², Dayane Quintino Vasconcelos ${ }^{2}$, Ana Carolina Albernaz Barbosa ${ }^{2}$, \\ Vinicius Nunes Silva ${ }^{1}$, Leonardo Santos Cardoso', Estevão Tavares de Figueiredo ${ }^{3}$
}

Coelho Júnior LG, Costa Junior WL, Vasconcelos DQ, Barbosa ACA, Almeida PRMN, Silva VN, Cardoso LS, Figueiredo ET. Paciente idosa portadora de doença aterosclerótica submetida à cineangiocoronariografia com achado incidental de origem e curso anômalos de artéria coronária esquerda: relato de caso / Patient elderly carrier of atherosclerotic disease undergoing coronary angiography with incidental finding of origin and course of anomalous left coronary artery: a case report. Rev Med (São Paulo). 2016 out.-dez.;95(4):175-8.

RESUMO: As anomalias das artérias coronárias são formas relativamente raras de malformação congênita acometendo aproximadamente $1 \%$ da população. O paciente portador de anomalia de artéria coronária pode apresentar-se assintomático por um longo período, ou, apresentar de forma súbita um infarto agudo do miocárdio, arritmias graves e até morte súbita. O diagnóstico baseado em história clínica e exame físico muitas vezes não é possível, pois não há alterações clínicas significativas; portanto o uso de exames de imagem é de suma importância, tais como cineangiocoronariografia, angiotomografia e ressonância de artérias coronárias. Relatamos um caso em que uma paciente idosa, hipertensa e tabagista, portadora de doença aterosclerótica apresentou angina instável e foi submetida à cineangiocoronariografia, a qual revelou de maneira incidental, origem e curso anômalos de artéria coronária esquerda.

Descritores: Anomalias dos vasos coronários; Doença das coronárias; Vasos coronários; Angiografia coronária; Revascularização miocárdica; Idoso.
ABSTRACT: Anomalies of the coronary arteries are relatively rare forms of congenital malformation affecting approximately $1 \%$ of the population. Patients affected by these anomalies may present themselves asymptomatic, or suffer of a sudden form of acute myocardial infarction, severe arrhythmia and even sudden death. The diagnosis based on clinical history and physical examination often can not be done, for there are no clinically significant changes; therefore the use of imaging is of utmost importance, as in coronary cineangiography, angiotomography and coronary arteries NMR. We report a case in which an elderly, hypertense and smoker patient, associated to atherosclerotic disease presented unstable angina and submitted to coronary cineangiography which revealed incidentally an anomalous origin and course of the left coronary artery.

Keywords: Coronary vessel anomalies; Coronary disease; Coronary vessels; Coronary angiography; Myocardial revascularization; Aged.

1 Acadêmico do Curso de Medicina, Faculdade Atenas, Paracatu-MG, Brasil. Emails: luilson-junior@hotmail.com, v_in_iciusnunes@hotmail. com, leocardosoptu@gmail.com, pedro.negreiros2@gmail.com.

2. Médico Residente de Clínica Médica, Faculdade Atenas, Paracatu-MG, Brasil. Emails: wesley.jr@hotmail.com, dayaneqv@hotmail.com, carolzinha_albernaz@hotmail.com, leocardosoptu@gmail.com.

3. Médico Cardiologista. Professor Titular do Departamento de Clínica Médica e Coordenador da Residência Médica em Clínica Médica da Faculdade Atenas, Paracatu- MG, Brasil. Email: estevaotavares@cardiol.br

Autor para correspondência: Luilson Geraldo Coelho Júnior. Rua Bernardo Caparucho, nº 30, apart. 04. Bela Vista. CEP: $38600-000$ Paracatu, MG, Brasil. Email: luilson-junior@hotmail.com 


\section{INTRODUÇÃO}

A s anomalias coronarianas são definidas por a origem, o curso e o término das artérias coronárias são anatomicamente desviados da normalidade e raramente encontrados na população geral. As anomalias coronarianas podem ocorrer em 1 a $5 \%$ dos pacientes submetidos à arteriografia coronariana, dependendo dos parâmetros de normalidade para definir uma variante anatômica ${ }^{1}$.

A maior razão para uma apropriada identificação das anomalias coronarianas é a de determinar suas propensões em desenvolver isquemia miocárdica, fixa ou dinâmica, arritmias e morte ${ }^{2}$. A origem e o trajeto proximal das artérias coronárias anômalas e suas ramificações são os principais fatores preditivos de gravidade ${ }^{3}$.

O paciente portador de anomalia de artéria coronária apresenta quadro clínico variável, o paciente pode permanecer assintomático por muitos anos, todavia, quando os sintomas estão presentes, ocorrem devido à isquemia miocárdica e podem manifestar-se sob a forma de angina, arritmias, síncope, infarto agudo do miocárdio e até mesmo morte súbita ${ }^{4}$.

A origem anômala das artérias coronarianas podendo apresentar um amplo espectro de formas clínicas, desde formas benignas consideradas variantes anatômicas assintomáticas até variantes potencialmente patológicas e evoluir de forma adversa; sendo assim, apresenta prognósticos variáveis; sendo assim, constitui um desafio diagnóstico ${ }^{4}$.

O objetivo deste trabalho é descrever um caso em que uma paciente idosa e portadora de doença aterosclerótica e síndrome coronariana aguda, que foi submetida à cineangiocoronariografia, a qual revelou de maneira incidental, origem e curso anômalos de artéria coronária esquerda.

\section{RELATO DE CASO}

Paciente de 74 anos, feminino, deu entrada na Unidade Coronariana com quadro de dor torácica anginosa constritiva, de início súbito há 30 minutos, com irradiação para membro superior esquerdo, com piora progressiva e sem alívio com o repouso. Ao exame: Taquicardíaca, dispneica, PA: 140x90mmHg; acianótica, afebril. Ausculta pulmonar e cardíaca sem alterações; realizado diagnóstico de angina instável de alto risco.

Nos antecedentes patológicos apresentava hipertensão arterial sistêmica em tratamento e relatava ser tabagista há aproximadamente 50 anos. Sem dados comemorativos sobre história familiar para doenças cardíacas.

Foi submetida à cineangiocoronariografia logo após o diagnóstico de angina instável (Figura 1), que evidenciou tronco único de ambas coronárias em seio coronariano direito, com a coronária direita (CD) exibindo lesão grave em terço médio, coronária esquerda $(\mathrm{CE})$ originandose no terço proximal da $\mathrm{CD}$, exibindo lesão grave em terço proximal. As artérias descendente anterior (DA) e circunflexa (CX) apresentavam apenas ateromatose difusa discreta.

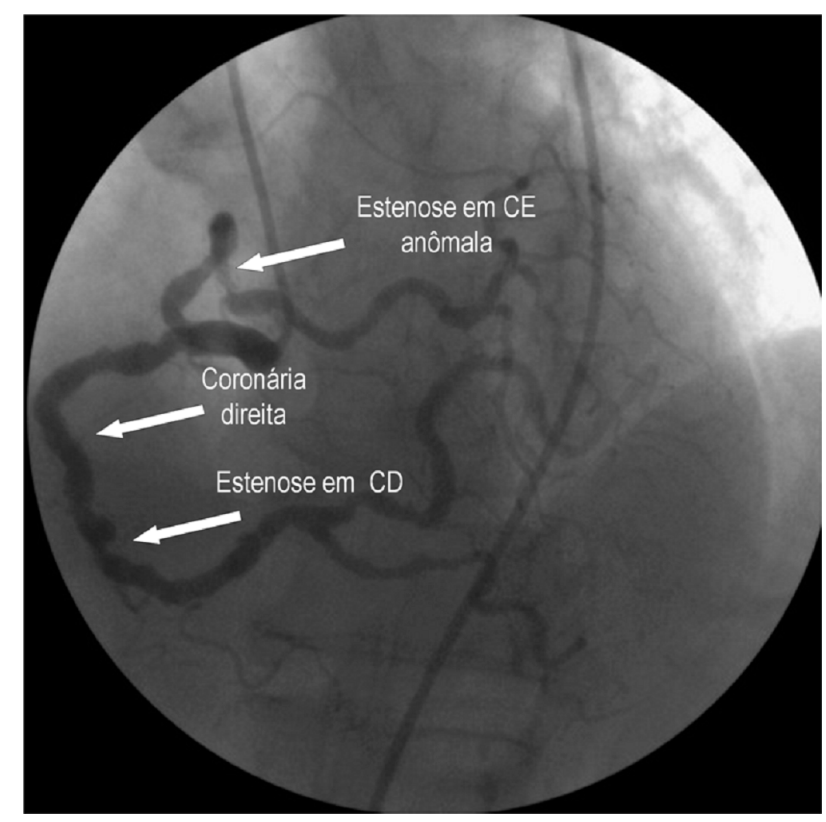

Figura 1. Cineangiocoronariografia

Uma vez optado por revascularização cirúrgica com circulação extracorpórea, a paciente foi submetida ao procedimento em novembro de 2011, com a realização de bypass da aorta ascendente para CX e CD, utilizando a veia safena magna, e anastomose da artéria torácica interna esquerda para a DA, com 62 min de pinçamento da aorta e 92 min de perfusão. Durante o ato operatório constatou-se que se tratava de origem anômala de coronária esquerda do tipo pré-pulmonar.

Houve boa tolerância do procedimento, ficando hospitalizada por quatro dias, sem intercorrências, com alta hospitalar em boas condições após quatro dias. Foi encaminhada ao ambulatório de cardiologia para seguimento, sem complicações após alta hospitalar.

\section{DISCUSSÃO}

Distúrbios embriogênicos ocorridos na terceira semana do desenvolvimento fetal originam anomalias das artérias coronárias. $\mathrm{O}$ coração origina-se inteiramente do mesoderma esplâncnico que forma o assoalho da cavidade pericárdica. Essa região é conhecida como área cardiogênica e origina um par de tubos endocárdicos que se unem para formar o tubo cardíaco primitivo ${ }^{5}$. As anomalias 
coronarianas decorrem de aspectos anatômicos diversos de acordo com sua origem, trajeto e distribuição.

O coração possui duas artérias coronárias, direita e esquerda, que se originam dos seios de Valsalva direito e esquerdo. $\mathrm{O}$ terceiro seio não origina nenhum vaso sanguíneo, sendo denominado de não coronariano ${ }^{6}$.

A artéria coronária esquerda normalmente sai do seio coronariano esquerdo e após uma distância de aproximadamente quatro $(04 \mathrm{~cm})$ bifurca-se em artéria circunflexa esquerda e descendente anterior; já a artéria coronária direita sai do seio coronariano direito e segue um trajeto posterior a artéria pulmonar, perto ao apêndice atrial direito, ao longo do sulco atrioventricular ${ }^{6,7}$. A artéria coronária direita irriga a parede do ventrículo direito; a artéria descendente anterior irriga a parede anterior e o septo interventricular; já a artéria circunflexa irriga a parede do ventrículo esquerdo ${ }^{8}$.

A nomenclatura anatômica de uma artéria coronária é dada de acordo com seu território de irrigação ${ }^{1}$. As anomalias coronarianas são classificadas de açodo com a classificação proposta por Angelini et al. ${ }^{1}$, em: anomalias de origem, de trajeto, anomalias intrínsecas e anomalias de terminação.

Existe uma outra classificação, que divide as anomalias coronarianas em hemodinamicamente significativas e não significativas. As anomalias coronarianas hemodinamicamente significativas englobam: as anomalias de origem com trajeto interarterial; com origem anômala na artéria pulmonar; as atresias e as fístulas congênitas ${ }^{8}$.

É de suma importância a realização de exames de imagem para o diagnóstico das anomalias coronarianas; haja vista, ser quase impossível o diagnóstico por meio da história clínica, exame físico e eletrocardiograma.

A anomalia congênita das artérias coronárias pode ter manifestação clínica variável, desde benigna até potencialmente grave, causando isquemia miocárdica, infarto, arritmias e morte súbita. A incidência varia de 0,3 a $1,5 \%$ em estudos de necropsia ou por cineangiocoronariografia ${ }^{3}$. Em um estudo de 1950 cineangiocoronariografias, a incidência encontrada foi de $5,6 \%{ }^{1}$. A documentação precisa do risco de isquemia para algumas dessas anomalias, utilizando testes de estresse físico convencional ou estudos intravasculares de Doppler fluxometria, é pouco preditiva, podendo falhar na detecção de anormalidades anatômicas significativas.

A angiotomografia computadorizada e a angioressonância são os métodos de imagem considerados padrões ouro para o diagnóstico das anomalias coronarianas. A angiografia já foi considerada o padrão ouro para o diagnóstico, todavia, a identificação do trajeto proximal pode ser difícil, além disso, a angiografia possui menor acurácia em comparação à angiotomografia computadorizada coronariana, esta possui uma acurácia de 55\%, como demonstrada no estudo de Schmitt et al. ${ }^{9}$. A angiografia é um exame ainda muito utilizado, sendo importante ressaltar seu uso para fins diagnósticos, na impossibilidade de realizar-se outro método de maior acurácia.

A origem anômala da CE do seio coronariano direito, como demonstrado no presente relato, pode relacionar-se a morte súbita em 59\% dos casos, precedida por atividade física em $81 \%$ dos eventos; o que não ocorreu com tal paciente, onde a anomalia foi descoberta de maneira ocasional (não suspeita) durante pesquisa de aterosclerose coronariana grave. Esta anomalia pode apresentar quatro trajetos (Figura 2): pré-pulmonar (anteriormente a artéria pulmonar); retroaórtico (posteriormente a aorta); interarterial (entra a aorta e a artéria pulmonar); e septal (abaixo do trato de saída do ventrículo direito). Todos os subtipos desta anomalia têm sido associados à morte súbita, mas a variação interarterial, que é o padrão mais comum, tem uma relação mais forte com sequelas catastróficas ${ }^{3}$.

Existem três formas de tratamento: observação e tratamento clínico; angioplastia com colocação de endoprótese e o tratamento cirúrgico ${ }^{1}$. O tratamento cirúrgico é o procedimento de escolha das anomalias coronarianas de origem e de trajeto. A revascularização cirúrgica com ponte vascular ou enxerto é uma técnica amplamente utilizada ${ }^{1}$.

Não há consenso na literatura para o manejo dos portadores da origem anômala de coronária esquerda da aorta. Nos indivíduos sintomáticos, deve-se optar pelo tratamento cirúrgico. As opções cirúrgicas são o implante de enxerto arterial ou venoso, o reimplante da artéria coronária anômala no seio coronariano anatômico, plastia do segmento intramural da artéria coronária e translocação do tronco da artéria pulmonar. Além disso, não existem dados que definam a melhor técnica cirúrgica.

Topaz et al. ${ }^{11}$ relatam o tratamento de cinco casos de lesões ateroscleróticas coronarianas com a angioplastia em pacientes com anomalias coronárias. Nos casos descritos, a avaliação da configuração do orifício coronário, angulação proximal, o trajeto do vaso e a localização da lesão estenótica foram essenciais para o sucesso do procedimento, sendo assim, a seleção adequada do cateterguia e o avanço do balão na porção proximal da artéria anômala são os maiores determinantes de sucesso.

É de suma importância evidenciar que em casos de obstruções ateroscleróticas associadas a anomalias de artérias coronárias, o estudo adequado do trajeto da artéria anômala é fundamental, haja vista, caso seja diagnosticado trajeto entre a artéria pulmonar e a artéria aorta, o tratamento cirúrgico é a opção preferencial para os casos eletivos.

Potencial Conflito de Interesses: Declaro não haver conflito de interesses pertinentes.

Fontes de Financiamento: O presente estudo não teve fontes de financiamento externas. 


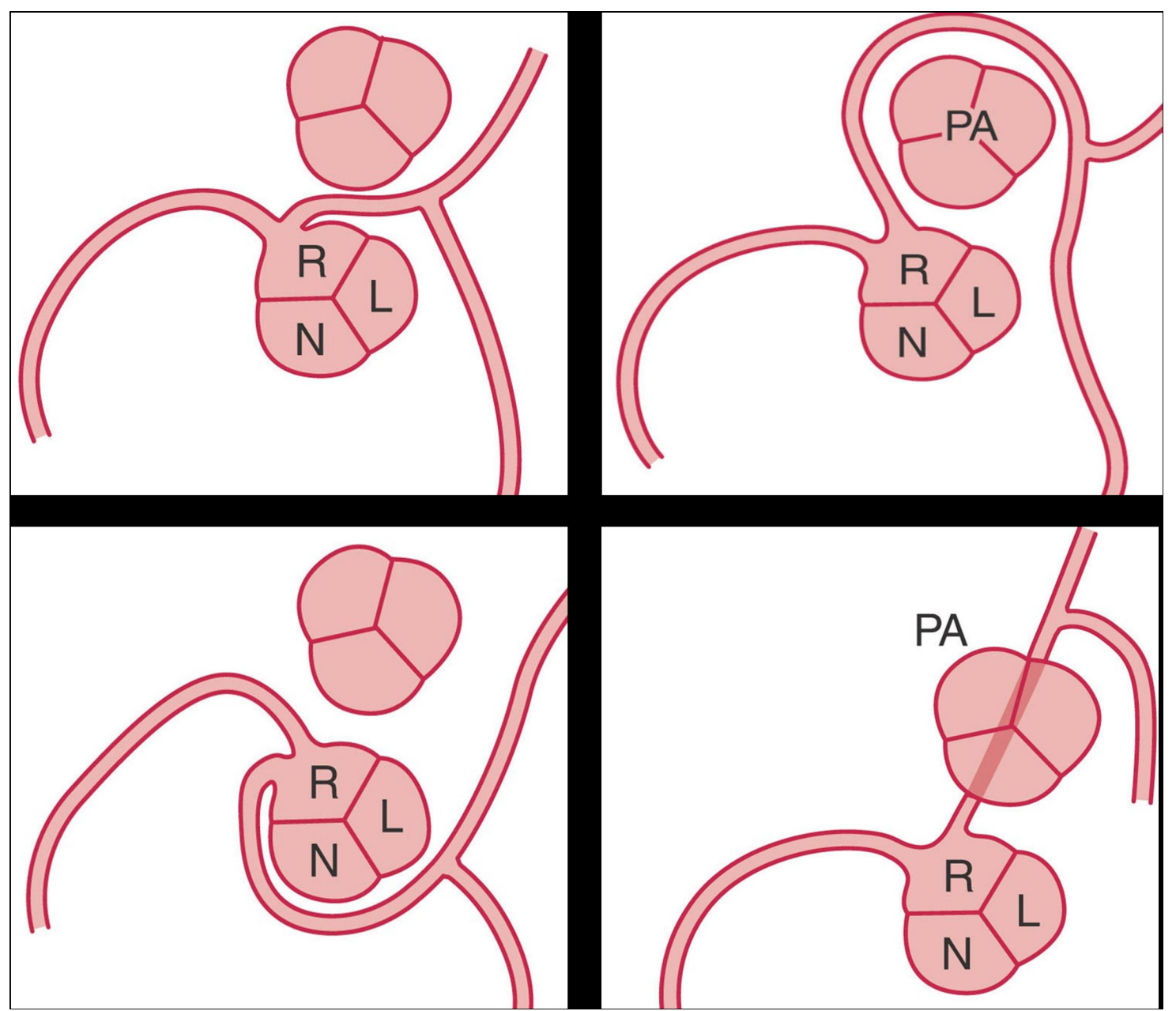

Figura 2. Variantes anatômicas da origem anômala de coronária esquerda da aorta (Adaptado de Popma $\mathrm{JJ}^{12}$ )

\section{REFERÊNCIAS}

1. Angelini P, Velasco JA, Flamm S. Coronary anomalies: incidence, pathophysiology, and clinical relevance. Circulation. 2002;105:2449-54. http://dx.doi.org/10.1161/01. CIR.0000016175.49835.57.

2. Eckart RE, Jones SO, Shry EA, et al. Sudden death associated with anomalous coronary origin and obstructive coronary disease in the young. Cardiol Rev. 2006;14:161-3. doi: 10.1097/01.crd.0000197965.99826.b2.

3. Veras FHAP, Victor EG, Saraiva LCR, et al. Origem anômala das artérias coronárias. Rev Bras Cardiol Invas. 2007;15(3):285-92. http://dx.doi.org/10.1590/S2179-83972007000300016.

4. Vieira C, Nabais S, Salgado A, Salomé N, Sousa P, Madureira AJ, et al. Origem coronária anómala: da suspeita à revascularização cirúrgica. Rev Port Cardiol. 2014;33(1):53. e1-e5. http://dx.doi.org/10.1016/j.repc.2013.07.010.

5. Fritzgerald MJT. Embriologia humana. São Paulo: Harper \& Row do Brasil; 1980. p.48.

6. Pfeiffer MET. Anomalia congênita de coronária relevância clínica, exercício e morte súbita. Rev DERC. 2013;19(4):114-8.

7. Rabelo DR, Barros MVL, Nunes MCP, et al. Angiotomografia coronariana multislice na avaliação da origem anômala das artérias coronarianas. Arq Bras Cardiol. 2012;98:266-72. http://dx.doi.org/10.1590/S0066-782X2012005000011.
8. Neves PO, Andrade J, Monção H. Artérias coronárias anômalas: o que o radiologista precisa saber. Radiol Bras. 2015;48(4):233-41. http://dx.doi.org/10.1590/01003984.2014.0004.

9. Chandra N, Bastiaenen R, Papadakis M, et al. Sudden cardiac death in young athletes: practical challenges and diagnostic dilemmas. J Am Coll Cardiol. 2013;61:1027-40. doi: 10.1016/j.jacc.2012.08.1032.

10. Hillis LD, Smith PK, Anderson JL, et al. 2011 ACCF/AHA Guideline for coronary artery bypass graft surgery; a report of the American College of Cardiology Foudation/American Heart Association Task Force on Practice Guidelines. Circulation. 2011;124:e652-735. http://dx.doi.org/10.1161/ CIR.0b013e31823c074e.

11. Topaz O, DiSciascio G, Goudreau E, Cowley MJ, Nath A, Kohli RS, et al. Coronary angioplasty of anomalous coronary arteries:notes on technical aspects. Cathet Cardiovasc Diagn. 1990;21:106-11. doi: 10.1002/ccd.1810210213.

12. Popma JJ. Angiografia coronariana e ultrassonografia intravascular. In: Libby P, Bonow RO, Mann DL, Zipes DP, editores. Braunwald tratado de doenças cardiovasculares. 8a ed. São Paulo: Elsevier; 2010. p.465-500. 